work suggesting that late onset diabetes in fact has the greater genetic component.

The chapter on genetic linkage succeeds admirably in dispelling much of the unnecessary complication which has deterred many ordinary investigators from work in this field; lod scores appear almost simple, and a full table of them is given in an appendix.

A crucial chapter introduces the valuable concept of the Bayesian approach to estimation of genetic risks, with clear examples of its use in X-linked and late onset dominant disorders. The importance of utilising risks appropriate to specific levels of carrier tests within the normal range is also discussed, though unfortunately an attempt is made to bring this into a cumbersome overall formula.

The final chapters are epidemiological in their approach. The methods and some of the pitfalls in searching for associations between genetic markers and diseases are outlined with emphasis on the HLA system and its associations, and on problems of significance levels when using multiple variables; useful tables of the main blood group and HLA associations are given.

Another valuable chapter compares the various approaches to analysis for a birth order or parental age effect and also stresses the importance and difficulty of providing comparable control data, giving useful tabular data extracted from the Registrar General's tables.

The last section, on changes in disease frequency, returns to classical epidemiology and gives clear examples of testing for frequency fluctuations and cyclical trends in congenital malformations. The use of CUSUM methods and Hewitts non-parametric method are particularly clearly illustrated.

The only general criticism that can be held against this valuable book is that a number of formulae are given without being clearly derived; this is perhaps inevitable in a book of this compactness, and it is surprising how fully the author has managed to treat most of the subjects in a small space. Though the price may seem high in relation to the number of pages, it is fully worth it, as this is a book which workers in medical genetics will not only wish to read thoroughly, but to use repeatedly for a wide variety of data.

Peter S. Harper

\section{Cancer Genetics.}

Edited by Henry Lynch. (Pp. Xv + 610;

Figures + Tables. $\$ 49 \cdot 50$.) Springfield, Illinois:

Charles C. Thomas. 1975.

Studies of the distribution of cancer in human populations suggest that given the right environment, 80 or $90 \%$ of cases would not occur. In the effort to find environmental causes for cancer which this suggestion has encouraged, the role of the genotype may be in danger of being neglected. The appearance of this book, for which 'Human cancer genetics' would be a more accurate title, is, therefore, timely as a reminder that genetic factors are undoubtedly involved in the aetiology of many neoplasms and that some of these relations (e.g. that of xeroderma pigmentosum to skin cancers) suggest models of carcinogenesis that may apply to other cancers also.

The editor is Professor of Preventive Medicine at Creighton University, Nebraska, and he and his colleagues there have written about half the book. The chapters fall into two groups, the first dealing principally with particular factors of genetic significance that affect liability to cancer-immunological status, histocompatibility, xeroderma pigmentosum, karyotype, and ethnic group-and the second with genetic and related aspects of particular neoplasmsespecially leukaemia and lymphoma, retinoblastoma, central nervous system tumours, and cancers of the colon, lung, skin, and breast.

Even for a multi-author work, the coverage of the subject matter is surprisingly variable, with more space devoted to Teter's histology-based classification of gonocytomas than to the whole fascinating subject of cancer incidence in different countries and in migrants and their descendants-which except for observations in Israel is hardly mentioned, despite the light it sheds on the relative importance of genotype and environment. Also, some of the chapters on particular neoplasms are naïve in their interpretation of the data presented. For example, the view that smoking is a simple autosomal recessive trait seems to be accepted as credible because of the results obtained when the observed frequency of smoking in the children of non-smokers is compared with the frequency expected on this hypothesis-results which arguably say more about the limitations of the methodology than about the determinants of smoking. By contrast, the book contains few if any comparisons between the kinds of observed and expected figures one would like to see-figures relating the frequency of specific neoplasms (e.g. in different groups of relatives of cases) to person-years of exposure, with the age distribution of these person-years taken into account in calculating the expected rates. Without some such figures to show how the risks vary according to family relationship, one cannot begin adequately to assess even the relative importance of genetic and environmental factors as causes of the familial clusters of cases that the editor and his colleagues describe, let alone the nature of whatever genetic causes there are. But though the book fails to give a fully balanced and rigorous appraisal of its subject and is not always as up to date as it could have 
been, it is still a useful guide to much of the literature on genetic aspects of human cancer.

IAN LECK

\section{Mutation Research. Problems, Results and Perspectives.}

By Charlotte Auerbach. (Pp. xviii +465 ;

Figures + Tables $110 \cdot 50$.) London: Chapman and Hall. 1976.

I find it very difficult to be objectively critical of the above book, since my own work on mutagenesis has been for some seven years directly, and subsequently indirectly, greatly influenced by Lotte Auerbach's ideas and outlook. In my opinion Professor Auerbach has written a unique and valuable book, in which is distilled the knowledge resulting from over three decades of work in mutagenesis. The 23 chapters provide a refreshing blend of historical perspective on early results, comparative viewpoints across the whole range of organisms studied, and critical incisiveness. Following early chapters dealing with the historical development of the subject, and the nature of mutations, $x$-ray and ultraviolet mutagenesis are dealt with in considerable detail. This is followed by several chapters on chemical mutagens, and others dealing with selected topics of continuing interest in mutagenesis such as completes and mosaics, mutagen specificities, spontaneous mutations, and instabilities. The final chapter covers the varied types of applied mutation research. As a reviewer my only regret is that the increasingly recognized importance of environmental chemical mutagens is not reflected in the mere 3-page coverage afforded in this book. One would certainly have welcomed Professor Auerbach's critical and authoritative appraisal of the various test systems currently being used to detect environmental mutagens.

It is to be hoped that this book will be available to all undergraduates taking final year genetics courses. It will be invaluable to all postgraduates and research workers involved in mutation research or testing, regardless of their particular organism or mutagen of choice. It is clearly printed and provided with numerous references to additional reading.

Colin H. Clarke

Haemoglobin: Structure, Function and Synthesis.

(British Medical Bulletin, Vol. 32, No. 3, September 1976.) Scientific Editor: D. J. Weatherall. (£3.00.) London: British Council. 1976.

Structure, Function and Synthesis of Haemoglobin is a suitable subject for the British Medical Bulletin. Britain has been foremost in its contribution to this field, and Dr Weatherall, the scientific editor has assembled a distinguished array of contributors 8 of whom everyone is recognised internationally as prominent expert.

M. F. Perutz contributes an introduction as well a survey of the structure of haemoglobin and of the structural alterations involved in the change fro oxy- to deoxyhaemoglobin. In the introduction Perutz asks what is it that makes the study of haem\& globin so absorbing. It is of course the fact that the haemoglobin is a two-way respiratory carrier, transporting oxygen from the lungs to the tissues and facilitating the return transport of $\mathrm{CO}_{2}$. It fulfils this dual function by a reversible change of its structure $s$ that the arterial form of haemoglobin has a hige affinity for oxygen and a low one for hydrogen, chloride ions, $\mathrm{CO}_{2}$, and organic phosphates with these relative affinities reversed in the intravenous fornt Perutz quotes Monod who conferred on haemoglobig the title of an 'honorary enzyme' calling the haem its active site, oxygen its substrate, and hydrogen ions its inhibitor. Organic phosphates which preferentially combine with the deoxy structure would then allosteric cofactors.

J. B. Kilmartin details the interaction of haemof globin with 2, 3-diphosphoglycerate, protons, an $\mathrm{CO}_{2}$, and J. M. Baldwin defines the Adair constants, oxygen equilibria, and co-operative interaction Against this background J. M. White describes the unstable haemoglobins where the delicately balance interactions between hydrophobic amino acis residues and the haem, as well as other intramolecula and subunit interactions, are disturbed. The dis tinctive role as a precipitating agent of the superio released in imbalanced oxygenation is described in what is perhaps the first fully understood molecula disease. A. J. Bellingham contributes a similax: analysis of the alteration in the oxygen affinity based on changes in molecular structure. A. May and E. R Huehns discuss the sickling process, both in vitro. and in vivo. It is not yet fully understood what happeng when the sickle cell haemoglobin forms monos directional crystals, but it is becoming quite clear that insoluble helical strands of molecules are formed and that these molecules interact with each other.

There is a very thorough survey of the genetics human haemoglobins assisted by what is now know of haemoglobin variants fusion and the products 06 crossing-over and deletions by A. Lang and P. Pu Lorkin with some very clear illustrations.

$R$. Williamson describes the measurement of globin genes in animals and man. The number human haemoglobin genes can now be expected to bo in the range of 8 to 10 of which 2 are $\alpha$ chain genes, 1 each a $\beta$ and a $\delta$ chain gene, and the rest to be divide between the $\gamma, \varepsilon$, and $\zeta$ chain genes.

N. J. Proudfoot and G. G. Brownlee describs 